\title{
Trombose venosa profunda de membros superiores. Estudo coorte retrospectivo de 52 casos
}

\author{
U pper-extremity deep vein thrombosis: a retrospective cohort study of 52 cases
}

\author{
Ricardo de Alvarenga Yoshida', M arcone Lima Sobreira², M ariangela Giannini ${ }^{2}$, Regina M oura ${ }^{3}$, \\ $\mathrm{H}$ amilton Almeida Rollo ${ }^{3}$, Winston Bonetti Yoshida4, Francisco $\mathrm{H}$ umberto de Abreu M affei ${ }^{5}$
}

\section{Resumo}

O bjetivo: Rever os fatores predisponentes e a evolução em série de casos de trombose venosa profunda dos membros superiores de nossa instituição.

M étodos: Cinqüenta e dois pacientes consecutivos, com trombose venosa profunda dos membros superiores ( 29 homens e 23 mulheres), idade média de 52,3 anos, documentados por mapeamento dúplex $(71,1 \%)$, flebografia $(11,1 \%)$ ou clinicamente $(15,6 \%)$, foram incluídos no presente estudo.

Resultados: As manifestações clínicas foram: dor no antebraço (24 casos - 46,1\%), dor no braço (27 casos - 51,9\%), edema do membro superior ( 45 casos - 86,5\%), dor à compressão do membro superior (36 casos - 70,2\%) e dor à movimentação do mesmo (32 casos - $61,7 \%$ ). O s principais fatores de risco foram: punção ou acesso venoso (20 casos - 39,1\%) e câncer ( 16 casos - 32,6\%). As veias envolvidas foram: umeral $(n=18)$, axilar $(n=27)$, subclávia $(n=15)$ e jugular $(n=11)$. A embolia pulmonar estava inicialmente presente em quatro casos (7,6\%). 0 tratamento inicial foi feito com heparina não-fracionada intravenosa $(64,3 \%)$, subcutânea $(16,7 \%)$, ou heparina de baixo peso molecular $(17,1 \%)$, seguido de varfarina. D oze pacientes morreram antes da alta, em função de causas não relacionadas à embolia pulmonar. Foram acompanhados os 40 pacientes restantes por período de 3 meses a 10 anos, sendo que dois morreram de causas não relacionadas à embolia pulmonar, um paciente desenvolveu seqüelas pós-trombóticas, como edema residual e limitações aos movimentos, e seis ficaram com discretos sintomas residuais (edema e dor).

C onclusões: A trombose venosa profunda dos membros superiores foi mais freqüente em pacientes submetidos a acessos venosos e com neoplasia em atividade. Comparando com dados da literatura, a evolução dos pacientes sob tratamento exclusivo com anticoagulantes foi, no mínimo, similar a outros tratamentos propostos.

Palavras-chave: trombose, trombose venosa, extremidade superior, anticoagulantes.

\begin{abstract}
O bjective: To review the predisposing factors and the evolution of upper-extremity deep vein thrombosis in a series of cases.

M ethods: Fifty-two consecutive patients ( 29 men and 23 women, mean age of 52.3 years) with upper-extremity deep vein thrombosis documented by duplex scan $(71.1 \%)$, phlebography $(11.1 \%)$ or clinically $(15.6 \%)$ were included in the study.

Results: Clinical manifestations were: forearm pain (24 cases $46.1 \%$ ), arm pain (27 cases - 51.9\%), upper limb edema (45 cases $86.5 \%$ ), pain to arm compression (36 cases - $70.2 \%$ ) and to arm movement (32 cases - 61.7\%). M ain risk factors were: vein puncture (20 cases $-39.1 \%)$, and cancer (16 cases - 32.6\%). D eep vein thrombosis involved humeral $(n=18)$, axillary $(n=27)$, subclavian $(n=15)$ and jugular ( $n=11$ ) veins. Pulmonary embolism was initially present in four cases (7.6\%). Initial therapy included unfractionated heparin, intravenous $(64.3 \%)$, subcutaneous $(16.7 \%)$ or low molecular weight heparin (17.1\%) administration, followed by warfarin. Twelve patients died before discharge from the hospital, due to causes not related to pulmonary embolism. The remaining 40 patients were followed-up for a period of 3 months to 10 years. Two died of causes not related to pulmonary embolism, one developed post-thrombotic sequels, such as residual edema and limitations to upper limb movement, and six had discrete residual symptoms (edema and pain).

Conclusions: Upper-extremity deep vein thrombosis was more frequent in patients submitted to venous access and with active neoplasia. The outcome of patients exclusively treated with oral anticoagulant was at least similar to other treatments proposed in the literature.
\end{abstract}

Key words: thrombosis, deep vein thrombosis, upper extremities, anticoagulants.

\footnotetext{
1. Médico residente de Angiologia e Cirurgia Vascular, Departamento de Cirurgia e Ortopedia, Faculdade de Medicina de Botucatu, Universidade Estadual Paulista (UNESP), Botucatu, SP

2. Médico(a) contratado(a), Departamento de Cirurgia e Ortopedia, Faculdade de Medicina de Botucatu, UNESP, Botucatu, SP

3. Doutor. Professor assistente, Departamento de Cirurgia e Ortopedia, Faculdade de Medicina de Botucatu, UNESP, Botucatu, SP

4. Livre-docente. Professor adjunto, Departamento de Cirurgia e Ortopedia, Faculdade de Medicina de Botucatu, UNESP, Botucatu, SP.

5. Professor titular, Departamento de Cirurgia e Ortopedia, Faculdade de Medicina de Botucatu, UNESP, Botucatu, SP
}

Artigo submetido em 09.06.05, aceito em 22.07.05. 
Estima-se que as tromboses venosas profundas de membros superiores (TVPM S) sejam responsáveis por 1 a $4 \%$ de todas as tromboses venosas profundas ${ }^{1,2}$. Embora as TVPM S sejam realmente menos freqüentes queas demembrosinferiores, suas complicaçõesembólicas não são menos graves 3,4 . Em revisão de 998 autópsias consecutivas, realizadas no $\mathrm{H}$ ospital das Clínicas daFaculdadedeM edicina de Botucatu (U N ESP) entre 1969 e 1976, foram encontrados 166 casos de tromboembolismo pulmonar (TEP), dos quais 7,9\% tiveram origem em veias dos membros superiores ${ }^{5}$. A longo prazo, esses pacientes podem também apresentar sintomas residuais graves nos membros acometidos ${ }^{6}$.

As TV PM S têm se tornado mais comuns nas últimas décadas, devido ao aumento do uso de cateteres para quimioterapia, diálise, nutrição parenteral e marcapassos ${ }^{7}$. Podem, também, ser provocadas por lesões decorrentes de estreitamentos causados por costel a cervical ou bandas fibrosas ou por neoplasias 8 . Além disso, podem estar relacionadas ao esforço, no qual ocorre aumento temporário da coagulação, lesões intimais microscópicas e estase venosa ${ }^{7,9}$. T ambém podem estar associadas a estados trombofílicos, com freqüência variável, de acordo com o estudo7,10.

Esses dados mostram a importância dessa doença, quenecessita dediagnóstico etratamento precoces para evitar as complicações. 0 presenteartigo éfruto deuma revisão dos casos de TVPM S tratados eacompanhados no Serviço de C irurgia V ascular de nossa instituição.

\section{Pacientes e métodos}

Foi feito um estudo observacional tipo coorte retrospectivo de série de casos de TVPMS ( $n=52)$, atendidos na $D$ isciplina de Cirurgia V ascular de nossa instituição no período compreendido entre 1992 e 2003. N o momento em que os pacientes com suspeita clínica de TVPM S eram atendidos em nosso hospital para diagnóstico e tratamento, eram preenchidos protocolos contendo dados demográficos, fatores de risco, comorbidades, medicações em uso, cirurgias realizadas, punções, cateterismos venosos, sinaisesintomas, método diagnóstico da TVPM S, veias acometidas, tratamento efetuado, complicações, embolia pulmonar (EP) e óbito. $\mathrm{N}$ ão havendo contra-indicações, os pacientes recebiam medicação anticoagulante e eram acompanhados no ambulatório de anticoagulação, geralmente por 3-6 meses, local onde se registrava sistematicamente, em outro protocolo, as doses de medicação, resultados de testes de coagulação e complicações.
Foi realizado um levantamento retrospectivo desses casos, juntando as informações desses dois registros em um protocolo final, feito no programa de banco de dados Epi-Info versão 3.2, complementando os dados com uma revisão dos prontuários e contato telefônico com os pacientes.

U tilizando os recursos do programa Epi-Info, foram feitas análises estatísticas descritivas dos dados demográficos, fatores de risco, comorbidades, mortalidade, extensão, tratamento e evolução da TVPM S.

\section{Resultados}

O s dados demográficos, tipos de caso, clínico ou cirúrgico, e comorbidades encontram-se na T abela 1. $\mathrm{N}$ ão houve diferença com relação a sexo e lado acometido. A média de idade foi $53,4 \pm 15,3$ anos (25-81 anos), sendo que $25 \%$ tinham menos de 42 anose $75 \%$ mais de 66 anos. A maioria dos pacientestinha doenças de tratamento clínico (57,6\%), em 32,7\% doenças cirúrgicas e 9,6\% foram TVPM S espontâneas. As comorbidades mais freqüentes foram: fumo, hipertensão arterial sistêmica (H AS), insuficiência cardíaca congestiva (ICC), obesidade, diabetes melito, nefropatia e 0 fato de os pacientes estarem acamados (T abela 1). As punções, cateterismos e 0 câncer foram os principais fatores de risco associados (T abela 2). No período estudado, não houve nenhum caso de trombose relacionada ao esforço. A presença de EP associada, confirmada por cintilografia de ventilação-perfusão, foi encontrada em $7,6 \%$ dos casos.

As manifestações clínicas mais relevantes foram: dor espontânea em braço $(51,9 \%)$ e antebraço $(46,1 \%)$ e edema do membro superior acometido $(86,5 \%)$ (Figura 1). N o exame clínico, a dor decorrente de compressão ou movimentação do membro superior estava presente em $70,2 \%$ e $61,7 \%$ dos pacientes, respectivamente.

0 diagnóstico foi confirmado pelo mapeamento dúplex (M D) em 71,1\%, e a flebografia foi utilizada em $11,1 \%$ dos casos, sendo que, em $15,6 \%$ dos mesmos, não foi realizado nenhum exame confirmatório, devido, principalmente, a limitações clínicas para transporte ou de punção venosa apresentadas por alguns pacientes. As veias mais acometidas foram as axilares, seguidas das subclávias, umerais ejugulares, bilateralmente, conforme Figura 2 .

0 tratamento inicial instituído para todos os casos foi, exclusivamente, a anticoagulação, em regime de 
Tabela 1 - D adosgeraisecomorbidadesdospacientescom trombose venosa profunda dos membros superiores

\begin{tabular}{llcc}
\hline Característica & & $\mathbf{n}$ & $\%$ \\
\hline Sexo & M asculino & 29 & 55,8 \\
Lado acometido & Feminino & 23 & 44,2 \\
& Direito & 25 & 49,0 \\
& Esquerdo & 27 & 51,0 \\
Tipo de caso & Cirúrgico & 30 & 57,6 \\
& Clínico & 17 & 32,7 \\
Comorbidades & Espontânea & 5 & 9,6 \\
& Fumo & 31 & 60,5 \\
& Hipertensão & 29 & 56,8 \\
& Acamado & 23 & 44,8 \\
& Cirurgia & 17 & 32,7 \\
& ICC & 14 & 26,8 \\
& O besidade & 12 & 24,0 \\
& Diabetes & 10 & 18,6 \\
& N efropatia & 9 & 17,1 \\
& AVC & 6 & 11,9 \\
& Embolia pulmonar & 4 & 8,0 \\
& Angina & 3 & 4,8 \\
& Fratura & 2 & 2,9 \\
& Infarto miocárdio & 1 & 2,4 \\
\hline
\end{tabular}

ICC = insuficiência cardíaca congestiva; $A V C=$ acidente vascular cerebral.

T abela 2 - Fatoresderisco paratrombosevenosa profunda

\begin{tabular}{lcc}
\hline Fatores de risco & $\mathbf{n}$ & $\%$ \\
\hline Punção ou cateterismo & 20 & 39,1 \\
Espontânea & 17 & 32,7 \\
Câncer & 16 & 32,6 \\
\hline
\end{tabular}

internação hospitalar. Foi ministrada heparina nãofracionada por via intravenosa (H N F-IV) em 64,3\% dos casos, HNF por via subcutânea (HNF-SC) em $16,7 \%$, ambas com doses controladas pelo tempo de tromboplastina parcial ativado (TTPA alvo entre 1,5 e $2,5)$, e heparina de baixo peso molecular (H BPM ) em $17,1 \%$ dos casos (doses detratamento por peso corporal indicadas pelos fabricantes, sem controle laboratorial), associados à varfarina em doses controladas pela razão normatizada internacional (RN I alvo entre 2,0 e 3,0). Em todos os casos, foi feito controle de plaquetas em dias alternados. As heparinas foram suspensas quando

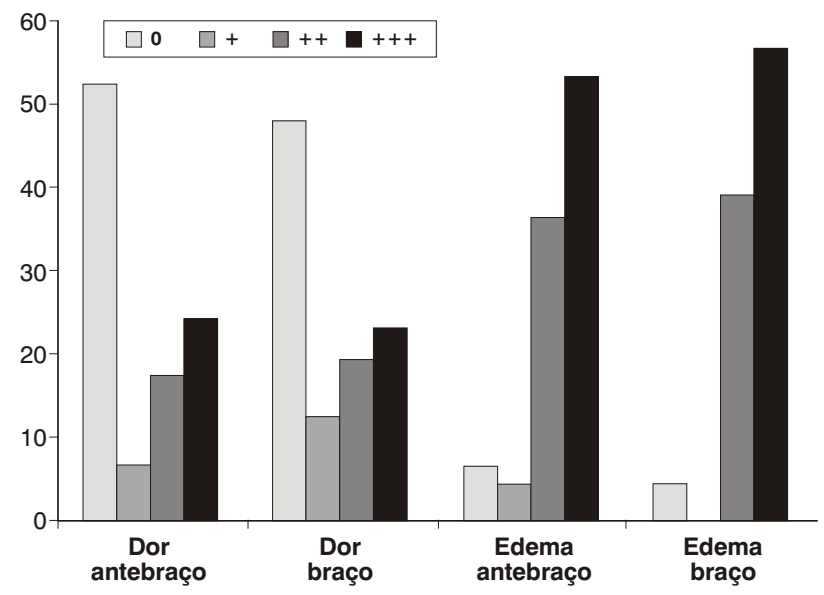

Figura 1 - F reqüências das manifestações clínicas dos pacientes

havia, pelo menos, 2 dias consecutivos de concomitância de tratamentos com RN I entre 2,0 e 3,0.

$\mathrm{N}$ a alta hospitalar, $65,4 \%$ dos pacientes referiram melhora clínica e 7,7\% permaneceram inalterados. D urantea internação, 23\% fal eceram devido às comorbidadesanteriormenteapresentadas. $\mathrm{N}$ enhum paciente teve recorrência de TEP ou qualquer complicação ligada ao tratamento anticoagulante no período de internação.

A duração do tratamento anticoagulante está na Tabela 3. A heparina foi ministrada, preferencialmente, entre 3-7 dias, e a varfarina entre 3-6 meses.

$\mathrm{N}$ a T abela 4, encontra-se o resumo da evolução de 40 pacientes que tiveram alta da enfermaria e foram acompanhados no ambulatório de anticoagulantes.

T abela 3 - D uração do tratamento anticoagulante

\begin{tabular}{llcc}
\hline Anticoagulante & D uração & $\mathbf{n}$ & $\%$ \\
\hline H eparina & $1-3$ dias & 6 & 15,0 \\
& $3-7$ dias & 25 & 62,5 \\
& 8-18 dias & 9 & 22,5 \\
Varfarina & & & \\
& 1-3 meses & 27 & 67,5 \\
& 3-6 meses & 9 & 22,5 \\
& 6-36 meses & 4 & 10,0 \\
\hline
\end{tabular}




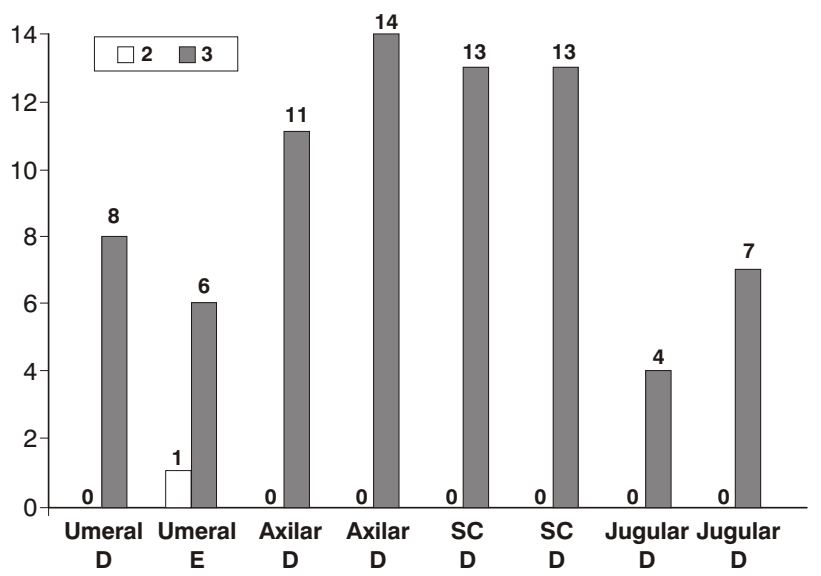

Figura 2 - Frequências das veias acometidas

T abela 4 - Evolução dos pacientes em longo prazo

\begin{tabular}{lcc}
\hline Sintomas & $\mathbf{1 - 3 6}$ meses & $\mathbf{1 - 1 0}$ anos \\
\hline Assintomáticos & 24 & 9 \\
Discretos & 6 & 6 \\
Limitantes & 1 & 1 \\
Perdeu seguimento & 7 & 15 \\
Ó bito & 2 & $?$ \\
\hline
\end{tabular}

D esses 40 casos, sete perderam seguimento e dois faleceram durante o acompanhamento no ambulatório de anticoagulação, por comorbidades provavelmente não relacionadas à $E P$, segundo descrito nos atestados de óbito. O s 31 pacientes remanescentes foram acompanhados no ambulatório de anticoagulação por 1 a 36 meses (média de 6 meses), sen do que 24 permaneceram assintomáticos, seis com sintomas discretos e um com sintoma limitante. Em 16 desses pacientes, foi possível obter informações sobre os sintomas remanescentes a longo prazo, em período de 1 a 10 anos (média de 5 anos), por meio de contato tel efônico, confirmando os seis pacientes com sintomas discretos, um com sintoma limitante e os nove restantes assintomáticos.

\section{D iscussão}

A TVPM S éuma doença multifatorial, relacionada principalmente a acessos venosos ${ }^{7}$ (os quais são predi- tores independentes dessa afecção) ${ }^{11}$ ea doenças mal ignas $8,12,13$. Nos pacientes do presente estudo, essas também foram as principais alterações associadas encontradas (T abela 1), principalmente nos pacientes cirúrgicos.

Alguns autores observaram prevalência expressiva de trombofilia nos pacientes com TV PM S, com razão de chances (odds ratio) de 4,09 nos pacientes com TVP idiopática, em relação aos casos de trombose de esforço ${ }^{10}$. M artinelli et al. ${ }^{14}$ observaram, em TVPM S primárias, razão de chances de 6,2 (95\%, intervalo de confiança 2,5 a 15,7) para o fator V Leiden; de 5 (95\%, intervalo de confiança 2 a 12) para protrombina G20210A; e 4,9 (95\%, intervalo de confiança 1,1 a 22) para deficiência de proteínas anticoagulantes, sendo que somentenesses casos há um aumento na incidência de TVPM S com o uso dos contraceptivos orais ${ }^{14}$. N os casos do presente estudo, essas determinações não foram feitas.

D o mesmo modo que para as TVPM I, o diagnóstico clínico é pouco sensível e específico. Logo, os sintomas, ossinaise osfatores de risco devem ser usados em conjunto para determinar a probabilidadeclínica de 0 indivíduo apresentar a afecção ${ }^{15}$. Assim, a suspeita clínica de TVPM S deve ser sempre levantada quando há aparecimento de dor e edema súbito dos membros superiores, principalmente nos casos em que as situações de risco já apontadas estão presentes. Outros achados que corroboram a suspeita seriam o desenvolvimento de circulação colateral visível e a dor exacerbando-se pela compressão e movimento muscular ${ }^{16}$. $\mathrm{N}$ a presente série, observou-se a predominância do edema $(86,5 \%)$ como principal sintoma, sendo a dor observada em cerca de $50 \%$ dos casos, acompanhada de circulação colateral, também em $50 \%$ deles (T abela 2). Burihan et al. ${ }^{16}$ observaram dor e edema em, respectivamente, 98 e $63 \%$ dos casos, e circulação colateral proeminente em $71 \%$ dos casos. Essas freqüências foram similares às observadas por outros autores ${ }^{17,18}$ em suas séries de casos. Além dos sintomas locais, sinais deEP podem preceder ou acompanhar o quadro clínico de TVPMS.

Para confirmação diagnóstica, na maioria dos nossos casos $(71,1 \%)$, foi realizado M D , o qual tem acurácia significativa para o diagnóstico da TV P 6 , variando de $80-100 \%$ de sensibilidade e especificidade ${ }^{12,19}$ (Figura 3). Trata-se do melhor método não-invasivo, tendo substituído a flebografia na avaliação do paciente com suspeita da doença. Apresenta muitas vantagens, 
como menor custo e ausência do risco do contraste iodado e da radiação. Sua acurácia melhora na medida em queos examinadoressetornam mais experienteseos aparelhos evoluem com melhor imagem e mais recursos. Aslimitações do M D , particularmenteem T V PM S, são: impossibilidade de compressão e dificuldade de visualização das veiasquetransitam abaixo deestruturas ósseas, ser examinador dependente e ter sensibilidade variável $^{20}$, particularmente nos membros superiores. N o entanto, o M D é o método preferível de diagnóstico, devendo ser utilizada a flebografia nos casos de dúvida no M D 7,12,21.

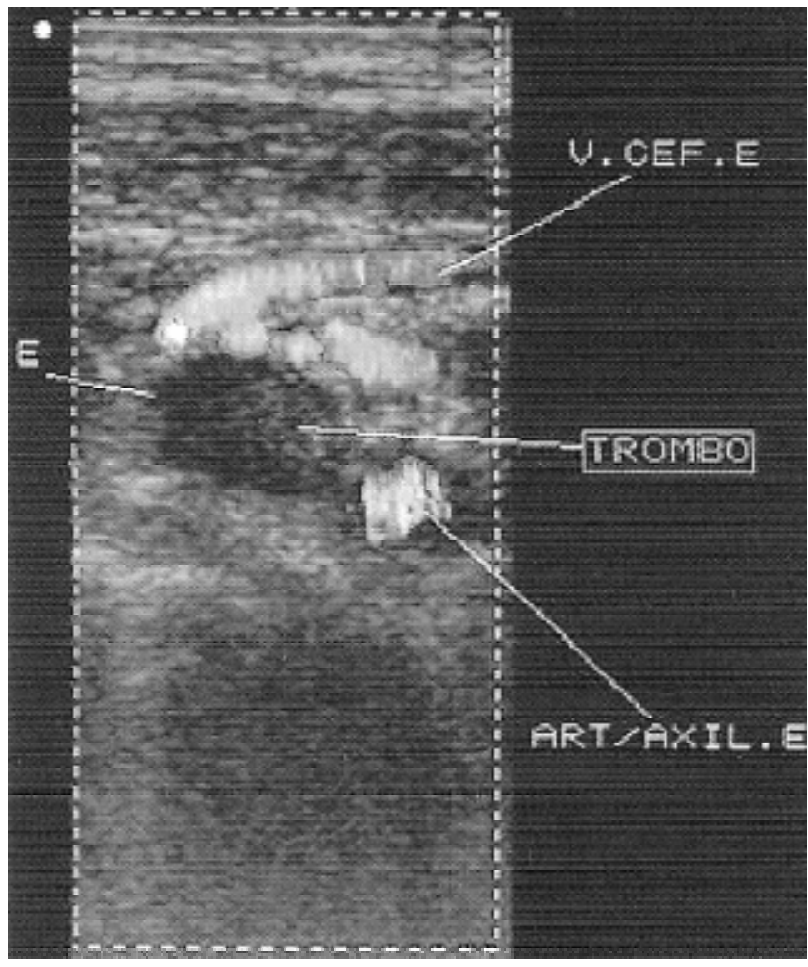

Figura 3 - Exemplo de mapeamento dúplex em paciente com trombose venosa profunda dos membros superiores acometendo veia axilar

A flebografia ainda é considerada o padrão-ouro dentre os exames complementares para o diagnóstico deTVP. A fal ha de enchimento do vaso pelo contraste determina a localização e a extensão do trombo ${ }^{22}$. É facilmente realizada nas situações em que há um cateter de flebotomia como causa dessa afecção, tracionando o cateter atéquea ponta fique localizada próximo ao ponto de sua entrada na veia e injetando 0 contraste iodado ${ }^{9}$. $\mathrm{N}$ o entanto, trata-se de exame invasivo, quepodetrazer desconforto para o paciente e provocar complicações, como reação de hipersensibilidade ao contraste e lesão endotelial com TVP em alguns pacientes ${ }^{23}$. Por esses motivos e pelo desenvolvimento do $M D$, a flebografia foi realizada na minoria de nossos dos pacientes (Figura 4).

$\mathrm{N}$ esta série, os exames de imagem para confirmação diagnóstica não puderam ser realizados em 15\% dos casos, por limitações de transporte apresentadas por alguns pacientes, optando-se por tratá-los sem confirmação.

A TVPMS pode causar complicações graves, como EP e insuficiência venosa crônica. Portanto, diante de suspeita clínica de EP, caracterizada por dispnéia, tosse, escarro hemoptóico e dor torácica, deve-se confirmar a hipótese diagnóstica com cintilografia pulmonar de ventilação e perfusão, queéum método simples, pouco invasivo e acurad $0^{24-26}$. $\mathrm{Na}$ série de 546 casos revistos por $\mathrm{H}$ ingorani et al. ${ }^{27}$, a mortalidade na TVPMS foi expressiva e devida, principalmente, às moléstias de base, como disfunções múltiplas de órgãos, e menos de complicações diretas da TVPM S, como a EP.

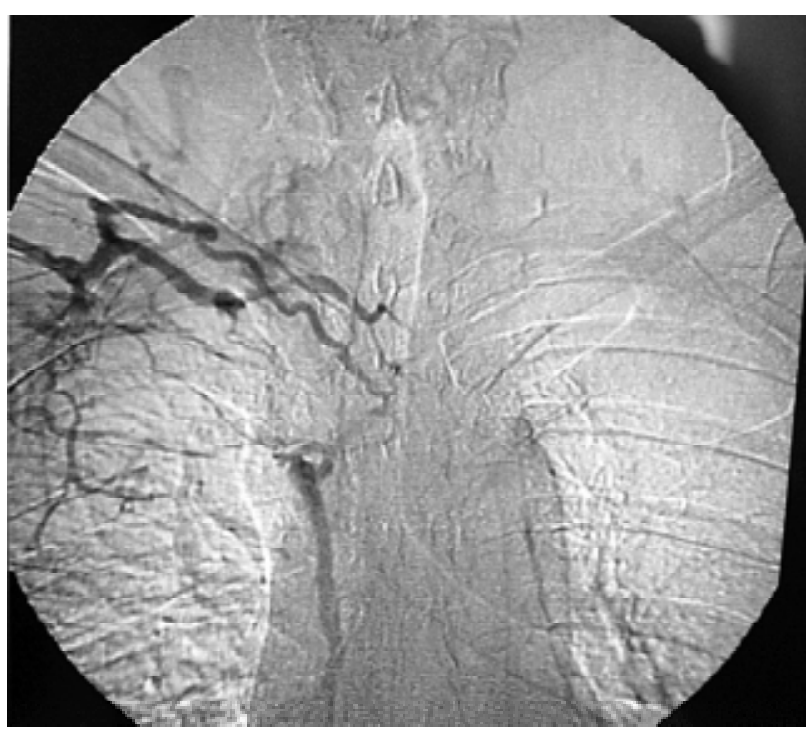

Figura 4 - Exemplo de flebografia de paciente com trombose venosa profunda dos membros superiores acometendo veia subclávia e veia cava superior 
0 tratamento da TVPM S deve ser introduzido precocemente, no sentido de evitar a EP e reduzir as complicações tardias de edema e limitações dos movimentos dosmembrossuperiores. Prandoni et al. encontraram EP em 0 a $12 \%$ dos casos de T VPM S, podendo chegar a 36\%, semel hante ao que ocorre na TVPM I. N o presenteestudo, encontramos $7,6 \%$ deEP sintomática confirmada, semel hante aos dados da literatura. 0 tipo de tratamento da T V PM S ainda é um assunto não totalmente definido. 0 tratamento anticoagulante impede o crescimento do trombo no interior da veia e previne $E P^{21,28,29}$. Recomenda-se a duração do tratamento inicial com H N F ou H BPM por, pelo menos, 5 dias. Embora não haja consenso estabelecido para TVPM S, a duração total do tratamento anticoagulante deve levar em conta os fatores de risco e a presença de $E P$, semelhante ao tratamento da TVPM I 21,30 . N esse sentido, os casos com fator causal transitório devem permanecer em anticoagulação por cerca de 3 meses; os pacientes com TVP espontânea e/ ou EP, por 6 meses; eoscasos deprimeira recorrência, por 12 a 36 meses. Os casos de segunda recorrência, presença de câncer ou trombofilia devem receber anticoagulantes perenemente ${ }^{31}$. N os pacientes com história familiar e pessoal de tromboses e em pacientes com TVPM S espontânea, é interessanteinvestigar a trombofilia ${ }^{6,14}$, poispodeestar presente em cerca de $26 \%$ desses casos $^{6}$. Entretanto, devido ao baixo risco deocorrêncianaT V PM S em geral e, inclusive, nos casos associados à trombofilia, outros autores sugerem queo tratamento por tempo prolongado ou ilimitado não se justificaria14.

Entretanto, sabemos que esse tipo de tratamento não impede, em todos os casos, complicações tardias, tais como: persistência do edema e desconforto, que limitam a função da extremidade. Em vista disso, autores têm preconizado tratamentos mais agressivos, os quais, em geral, foram utilizados em pequenas séries decasos, sem terem evidência científica comprovada 7,9 .

Assim, Adams et al. ${ }^{32}$ sugeriram a trombectomia venosa associada à descompressão venosa (retirada da costela, secção muscular ou de banda fibrosa), queteria melhor indicação nos casos com história clínica menor que 5 dias e imagem flebográfica de trombo recente. U ma al ternativa, nessa situação, seria o uso de fibrinolíticos in situ 33 , seguido de descompressão venosa, quando a flebografia mostrar essa alteração ${ }^{34}$. Por outro lado, já foram observados resultados promissores com anticoagulação associada à descompressão, sem trombectomia ${ }^{35}$, para oscasosacima referidos. Recentemente, com avançostecnológicos dos procedimentosendovasculares (balões, stentse endopróteses), é possível que essestratamentosvenham a ocupar um espaço maior no tratamento da TVPM S. A T abela 5 mostra, comparativamente, nossos resultados com os de outros autores, em relação aos sintomas residuais relacionados ao tipo de tratamento. Em nossa série de casos, esses não se mostraram elevados e foram semelhantes aos de Burihan et al. ${ }^{16}$ e de $\mathrm{H}_{\text {eron }}{ }^{36}$.

T abela 5 - C Comparação dos resultados de vários autores no tratamento da trombose venosa profunda dos membros superiores

\begin{tabular}{|c|c|c|c|c|}
\hline Autores & Tratamento & Assintomático & $\begin{array}{l}\text { Sintomas } \\
\text { D iscretos }\end{array}$ & Limitação \\
\hline $\begin{array}{l}\text { Burihan et al. }{ }^{16} \\
n=52\end{array}$ & Anticoagulante & $40 \%$ & $34 \%$ & - \\
\hline $\begin{array}{l}\text { Yoshida et al. } \\
n=52\end{array}$ & Anticoagulante & $77 \%$ & $15 \%$ & $2,5 \%$ \\
\hline $\begin{array}{l}\text { H eron et al. }{ }^{36} \\
n=54\end{array}$ & Anticoagulante & $50 \%$ & $37 \%$ & $13 \%$ \\
\hline $\begin{array}{l}\text { Zimmermann et al. }{ }^{37} \\
n=13\end{array}$ & U rokinase sistêmica & - & $50 \%$ & - \\
\hline $\begin{array}{l}\text { Sheeran et al. }{ }^{38} \\
n=9\end{array}$ & U rokinase local & - & $55 \%$ & - \\
\hline $\begin{array}{l}M \text { achleder }{ }^{39} \\
n=36\end{array}$ & $\begin{array}{l}\text { U rokinase local + } \\
\text { cirurgia de descompressão }\end{array}$ & $86 \%$ & - & - \\
\hline
\end{tabular}


C oncluímos, então, com base nos achados deste estudo, que as TVPM S foram mais freqüentes em pacientes submetidos a acessos venosos e com neoplasia em atividade. Comparando com dados da literatura, a evolução dos pacientes sob tratamento exclusivo com anticoagulantes foi, no mínimo, similar a outros tratamentos propostos.

\section{R eferências}

1. H ulbert S, Rutherford R. Subclavian-axillary vein thrombosis. In: Rutherford R, editor. V ascular surgery. Volume 1. Philadelphia: W. B. Saunders C 0.; 2000. p. 208.

2. Arcelus JI, Caprini JA, M onreal M, Suarez C, GonzalezFajardo J. The management and outcome of acute venous thromboembolism: a prospective registry including 4011 patients. J V asc Surg. 2003;38:916-22.

3. Horattas $M C, W$ right $D J$, Fenton $A H$, et al. Changing concepts of deep venous thrombosis of the upper extremityreport of a series and review of the literature. Surgery. 1988;104:561-7.

4. M onreal M, Raventos A, Lerma R, Ruiz J, Lafoz E, Alastrue A, Llamazares]F. Pulmonary embolism in patients with upper extremity DVT associated to venous central lines- a prospective study. Thromb H aemost. 1994;72:548-50.

5. $M$ affei $F$, FaleirosA, V enezian $C$, et al. C ontribuição ao estudo da incidência e anatomia patológica do tromboembolismo pulmonar em autópsias. Rev Assod M ed Bras. 1980;26:7-10.

6. Prandoni $P$, Polistena $P$, Bernardi $E$, et al. U pper-extremity deep vein thrombosis. Risk factors, diagnosis, and complications. Arch Intern M ed. 1997;157:57-62.

7. Joffe HV, Goldhaber SZ. U pper-extremity deep vein thrombosis. Circulation. 2002;106:1874-80.

8. M onreal M, Alastrue A, Rull M , et al. U pper extremity deep venous thrombosis in cancer patients with venous access devices - prophylaxis with a low molecular weight heparin (Fragmin). Thromb H aemost. 1996;75:251-3.

9. Yoshida WB. Trombose venosa profunda dos membros superiores. In: M affei FH A, Lastoria S, Yoshida W B, Rollo $H A$, editores. D oenças vasculares periféricas. Volume 2 . Rio de Janeiro: M edsi; 2002. p. 1433-1440.

10. Heron $E$, Lozinguez 0 , Alhenc-Gelas $M$, Emmerich J, Fiessinger JN. H ypercoagulable states in primary upperextremity deep vein thrombosis. Arch Intern Med. 2000;160:382-6.

11. Joffe HV, Kucher N, Tapson VF, Goldhaber SZ. U pperextremity deep vein thrombosis: a prospective registry of 592 patients. Circulation. 2004;110:1605-11.

12. Baarslag $H J$, van Beek EJ, Koopman MM, Reekers JA. Prospective study of color duplex ultrasonography compared with contrast venography in patients suspected of having deep venous thrombosis of the upper extremities. Ann Intern M ed. 2002;136:865-72.
13. Baarslag $\mathrm{HJ}, \mathrm{Koopman} M \mathrm{M}, \mathrm{H}$ utten $\mathrm{BA}$, et al. Long-term follow-up of patients with suspected deep vein thrombosis of theupper extremity: survival, risk factorsand post-thrombotic syndrome. Eur J Intern M ed. 2004;15:503-7.

14. M artinelli I, Battaglioli T, Bucciarelli P, Passamonti SM, $M$ annucci PM. Risk factors and recurrence rate of primary deep vein thrombosis of the upper extremities. Circulation. 2004;110:566-70.

15. Rollo H, Fortes V, Fortes J r A, Yoshida W, Lastoria S, M affei $F$. Abordagem diagnóstica dos pacientes com suspeita clínica de trombose venosa profunda dos membros inferiores. J V asc Br. 2005;4:79-92.

16. Burihan $E$, deFigueiredo LF, M iranda J F F. U pper-extremity deep venousthrombosis: analysis of 52 cases. C ardiovasc Surg. 1993;1:19-22.

17. $H$ ingorani $A$, Ascher $E$, Lorenson $E$, et al. Upper extremity deep venous thrombosis and its impact on morbidity and mortality rates in a hospital-based population. J V asc Surg. 1997;26:853-60.

18. $M$ ariel, LevesqueH, C ailleuxN , et al. [D eep venousthrombosis of the upper limbs. Apropos of 49 cases]. Rev M ed Interne. 1998;19:399-408.

19. Prandoni $P$, Lensing AW, Cogo A, et al. The long-term clinical course of acute deep venous thrombosis. Ann Intern M ed. 1996;125:1-7.

20. M ustafa BO, Rathbun SW, Whitsett TL, Raskob GE. Sensitivity and specificity of ultrasonography in the diagnosis of upper extremity deep vein thrombosis: a systematic review. Arch Intern M ed. 2002;162:401-4.

21. Prandoni P, Bernardi E. U pper extremity deep vein thrombosis. Curr O pin Pulm Med. 1999;5:222-6.

22. Baarslag $H J$, van Beek EJ, Tijssen JG, van Delden $O M$, Bakker AJ, Reekers JA. D eep vein thrombosis of the upper extremity: intra- and interobserver study of digital subtraction venography. Eur Radiol. 2003;13:251-5.

23. Rollo H A, M affei F, Lastoria S, Yoshida W, Castiglia V. U so rotineiro de flebografia no diagnóstico de trombose venosa profundademembrosinferiores. CirV ascAngiol. 1986;2:7-12.

24. PIO PED. Value of the ventilation/perfusion scan in acute pulmonary embolism. Results of the prospective investigation of pulmonary embolism diagnosis (PIO PED ). The PIO PED Investigators. JAM A. 1990;263:2753-9.

25. Stein PD, H ull RD, Saltzman HA, Pineo G. Strategy for diagnosis of patientswith suspected acutepulmonary embolism. Chest. 1993;103:1553-9.

26. Q ueluzTH, Yoo H H. Tromboembolia pulmonar: diagnóstico e tratamento clínico. In: M affei FHA, Lastoria S, Yoshida W B, Rollo $H A$, editores. D oenças vasculares periféricas. Volume 2. Rio de Janeiro: M edsi; 2002. p. 1453-1466.

27. H ingorani $A, A$ scher $E, M$ arkevich $N$, et al. Risk factors for mortality in patientswith upper extremity and internal jugular deep venous thrombosis. J V asc Surg. 2005;41:476-8.

28. Ellis M H , M anor Y, W itz M . Risk factors and management of patients with upper limb deep vein thrombosis. Chest. 2000;117:43-6.

29. M ustafa S, Stein PD, Patel KC, Otten TR, Holmes R, Silbergleit $A$. U pper extremity deep venousthrombosis. $C$ hest. 2003;123:1953-6. 
30. Savage KJ, W ells PS, Schulz V, et al. O utpatient use of low molecular weight heparin ( $D$ alteparin) for the treatment of deep vein thrombosis of the upper extremity. Thromb $\mathrm{H}$ aemost. 1999;82:1008-10.

31. M affei $F H$, Rollo H A, LastoriaS. T rombosevenosa profunda dos membros inferiores: tratamento clínico. In: M affei FHA, L astoriaS, YoshidaW B, Rollo H A, editores. D oençasvasculares periféricas. Volume2. Rio de Janeiro: M edsi; 2002. p. 14071426.

32. Adams Jr CZ, Vincent GS, Vermilion B. Primary effort thrombosis: treatment options in acute and chronic disease case reports. V asc Surg. 1991;4:234-9.

33. C astaneda F, Li R, Y oung K, Swischuk JL, Smouse B, B rady $T$. C atheter-directed thrombolysis in deep venousthrombosis with use of reteplase: immediate results and complications from a pilot study. J V asc Interv Radiol. 2002;13:577-80.

34. M olina JE. Surgery for effort thrombosis of the subclavian vein. J Thorac Cardiovasc Surg. 1992;103:341-6.

35. Ross D B. Axillary-subclavian vein occlusion. In: R utherford RB, editor. V ascular surgery. V olume 1. Philadel phia: W. B. Saunders C o.; 1984. p. 385-393.

36. H eron $E$, L ozingues $0, E$ mmerich J, et al. Long-term sequelae of spontaneous axillary-subclavian venous thrombosis. Ann Intern M ed. 1999;108:1-9.
37. Zimmermann R, M orl H, H arenberg J, Gerhardt $P$, Kuhn $H M, W$ ahl $P$. U rokinase therapy of subclavian-axillary vein thrombosis. Klin W ochenschr. 1981;59:851-6.

38. Sheeran SR, H allisey M J M urphy TP, Faberman RS, Sherman S. Local thrombolytic therapy as part of a multidisciplinary approach to acute axillosubclavian vein thrombosis (PagetSchroetter syndrome). J Vasc Interv Radiol. 1997;8:253-60.

39. M achleder HI. Evaluation of a new treatment strategy for Paget-Schroetter syndrome: spontaneous thrombosis of the axillary-subclavian vein. J V asc Surg. 1993;17:305-17.

\section{Correspondência:}

Ricardo de Alvarenga Yoshida

D epartamento de Cirurgia e 0 rtopedia

Faculdade de M edicina de Botucatu - U NESP

CEP 18618-970 - Botucatu, SP

Tel.: (14) 3811.6269

E-mail: ricardoyoshida@gmail.com 